\title{
Correction: Keratinocyte differentiation promotes ER stress-dependent lysosome biogenesis
}

Sarmistha Mahanty', Shruthi Shirur Dakappa', Rezwan Shariff², Saloni Patel', Mruthyunjaya Mathapathi Swamy², Amitabha Majumdar ${ }^{2}$ and Subba Rao Gangi Setty $\mathbb{1 D}^{1}$

\section{Correction to: Cell Death and Disease https://doi.org/10.1038/s41419-019-1478-4; published online 19 March 2019}

Following publication of this article, the authors realized there was an error in Fig. $2 b$ that needed correction. The TFEB panel of Fig. $2 b$ (total lysate) appears to be the same as the TFEB panel of Fig. 2e (cytosolic fraction); the TFE3 panels of Fig. 2b (total lysate) appear to be the same as the TFE3 panels of Fig. 2e (cytosolic fraction) which happened during image assembly. The corrected figure is provided below. This error did not impact the scientific conclusions of the article. We apologize for any inconvenience to the readers.

This has been corrected in both the PDF and HTML versions of the Article.

Published online: 03 October 2019 
a Epidermal skin sample

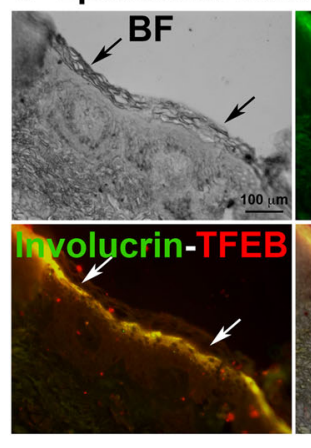

d Control

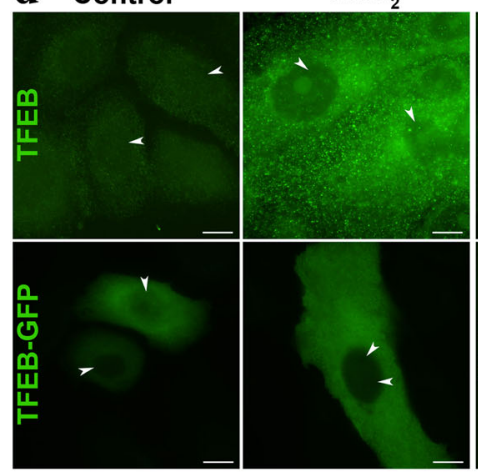

e

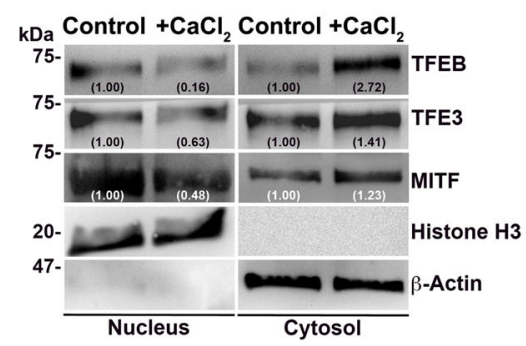

b
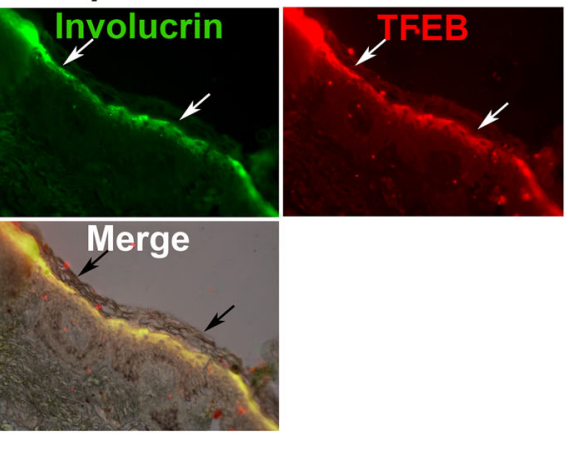

Control $+\mathrm{CaCl}_{2}$
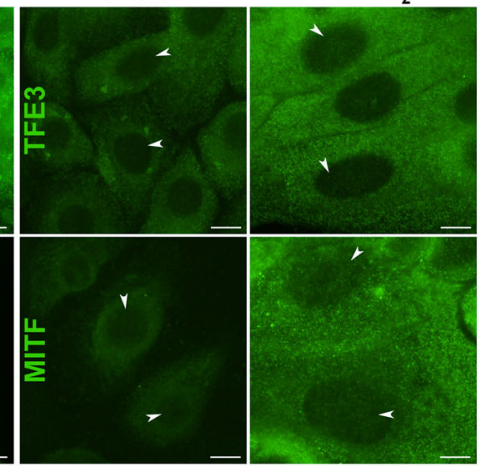

c
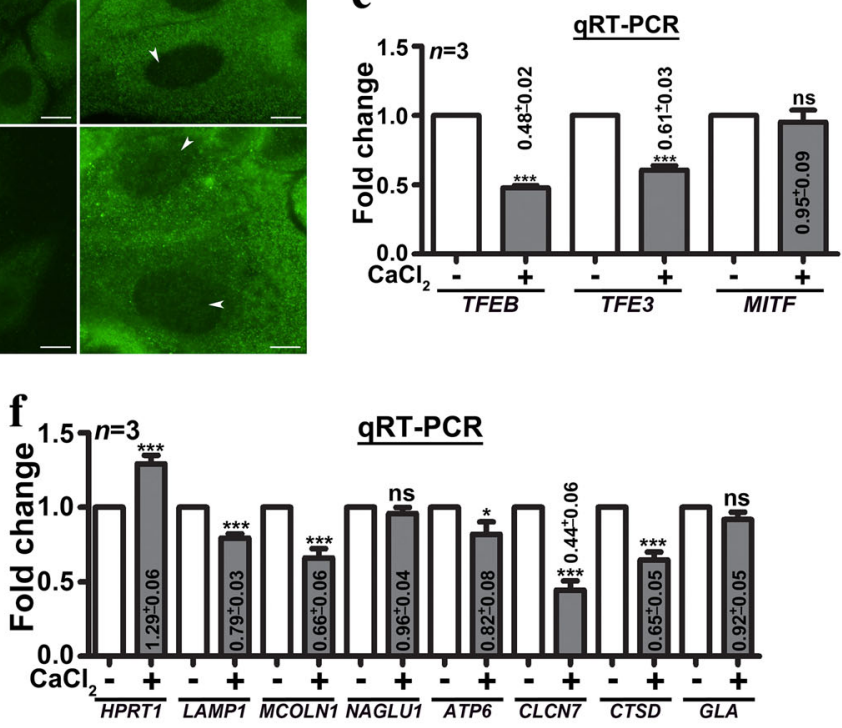

Fig. 2 a BF and IFM of epidermal skin sample that was immunostained for involucrin and TFEB. Black arrows point to the cornified skin layer and white arrows indicate the involucrin and TFEB-positive layer. Scale bar, $100 \mu \mathrm{m}$. b, c Immunoblotting and qRT-PCR analyses of MiT/TFE TFs (TFEB, TFE3, and MITF). $\mathbf{d}$, e IFM and nuclear fractionation analyses of keratinocytes for the localization of TFEB-GFP or endogenous MiT/TFE TFs. Arrowheads point to the localization of TFs to the nucleus. Scale bars, $10 \mu \mathrm{m}$. In $\mathbf{b}$ and $\mathbf{e}$, the fold change in protein levels is indicated after normalization with respective loading controls. $\mathbf{f}$ qRT-PCR analysis of various lysosome biogenesis genes. In $\mathbf{c}$ and $\mathbf{f}$, the fold change (mean \pm s.e.m.) in gene expression is indicated $(n=3) .{ }^{*} p \leq 0.05 ;{ }^{* *} p \leq 0.001$ and $\mathrm{ns}$, not significant 